\title{
Deep Levels Induced by CdTe/ZnTe Quantum Dots
}

\author{
E. Zielony ${ }^{a}$, E. Placzek-Popko ${ }^{a}$, A. Roznicka ${ }^{a}$, Z. Gumienny ${ }^{a}$, J. Szatkowski ${ }^{a}$, \\ P. Dyba ${ }^{a}$, W. Pacuski ${ }^{b, c}$, C. Kruse ${ }^{c}$, D. Hommel ${ }^{c}$ And M. Guziewicz ${ }^{d}$ \\ ${ }^{a}$ Institute of Physics, Wrocław University of Technology, Wybrzeże Wyspiańskiego 27, 50-370 Wrocław, Poland \\ ${ }^{b}$ Institute of Experimental Physics, University of Warsaw, Hoża 69, 00-681 Warsaw, Poland \\ ${ }^{c}$ Institute of Solid State Physics, University of Bremen, Postfach 330 440, D-28334 Bremen, Germany \\ ${ }^{d}$ Institute of Electron Technology, al. Lotników 32/46, 02-668 Warsaw, Poland
}

\begin{abstract}
The electrical properties of the CdTe/ZnTe quantum dot system have been analyzed to identify deep-level defects related with the presence of quantum dots. The capacitance-voltage $(C-V)$ and deep level transient spectroscopy measurements were used to investigate the samples. A reference ZnTe sample (without dots) was also studied for comparison. Both samples were grown by molecular beam epitaxy technique on the $n$-type GaAs substrate. The quantum dots were formed by a Zn-induced reorganization of a thin CdTe layer. The presence of quantum dot formation was confirmed by micro-photoluminescence measurements. The deep level transient spectra for both samples are complex. In order to characterize individual contributions to the deep level transient spectra the latter have been simulated by separated Gaussian components [1]. The results of the deep level transient spectroscopy measurements yield the conclusion that the same defects are present in both materials but there is an increased concentration of the defects in the quantum dot structures. No deep level associated directly with the quantum dot confinement has been identified.
\end{abstract}

PACS: 73.61.Ga, 73.21.La, 73.20.Hb

\section{Introduction}

Quantum dots have recently found applications in microelectronics and optoelectronics [1-3]. The electronic properties, which are important for the design and understanding of a variety of devices, can only be determined by electrical methods such as space charge capacitance techniques. The capacitance-voltage $(C-V)$ measurements and deep level transient spectroscopy (DLTS) method have been used with considerable success to study electronic properties of quantum dots [4-6].

An important progress has been recently achieved in fabrication of tellurium-based II-VI photonic structures. Zinc telluride ( $\mathrm{ZnTe}$ ) has been long considered as a very promising material for green optoelectronics. With respect to applications in green optoelectronics the $\mathrm{CdTe} /$ ZnTe quantum dot systems are particularly interesting. For this argument, the basic knowledge on exact band diagram of the CdTe/ZnTe QD system is of crucial importance.

In this paper the electronic properties of $\mathrm{CdTe} / \mathrm{ZnTe}$ QD structures have been studied using $C-V$ and DLTS measurements. A reference sample without quantum dots has been analyzed for comparison. The DLTS signal peaks have been simulated by separated Gaussian components [7]. The apparent activation energy, its broadening and capture cross-section were used as the fitting parameters.

\section{Experimental details}

Two types of samples with the same layer structure were processed by molecular beam epitaxy method: a ref- erence sample without quantum dots and a sample with quantum dots. The quantum dots were formed by a Zn-induced reorganization of a thin CdTe layer, similar to the formation of CdSe-QDs in ZnSe matrix [8]. The QD sample consists of $62 \mathrm{~nm}$ thick undoped ZnSe spacer deposited on the $n$-type GaAs substrate, $1040 \mathrm{~nm}$ of undoped ZnTe buffer, $2 \mathrm{~nm}$ thick CdTe layer which transforms to CdTe QDs and $100 \mathrm{~nm}$ of undoped ZnTe cap. The presence of quantum dots was confirmed by sharp peaks observed in micro-photoluminescence spectra (Fig. 1). The reference sample has the same structure but without the cap layer and quantum dots.

The Schottky $\mathrm{Ti}$ contacts were processed on top of the specimens and the ohmic $\mathrm{Au} / \mathrm{Ge} / \mathrm{Ni}$ contacts were completed on their backside. The capacitance-voltage $(C-V)$ measurements were performed on the lock-in based DLS-82E System (SEMITRAP, Hungary).

\section{Results and discussion}

In Fig. 1 the micro-photoluminescence plot measured for the QD sample, at a temperature of $2 \mathrm{~K}$, is shown. Sharp peaks observed in the micro-photoluminescence spectrum confirm the presence of quantum dots.

Figure 2 presents the $C-V$ plots for the referenceand QD sample measured at room temperature. A characteristic step, which is normally seen in the $C-V$ characteristics for samples containing quantum dots, does not appear in this case. This step is related to the accumulation of carriers in the dots states and can be visible solely if the capacitance of quantum dots predominates. It seems that this condition is not fulfilled in the studied samples. 


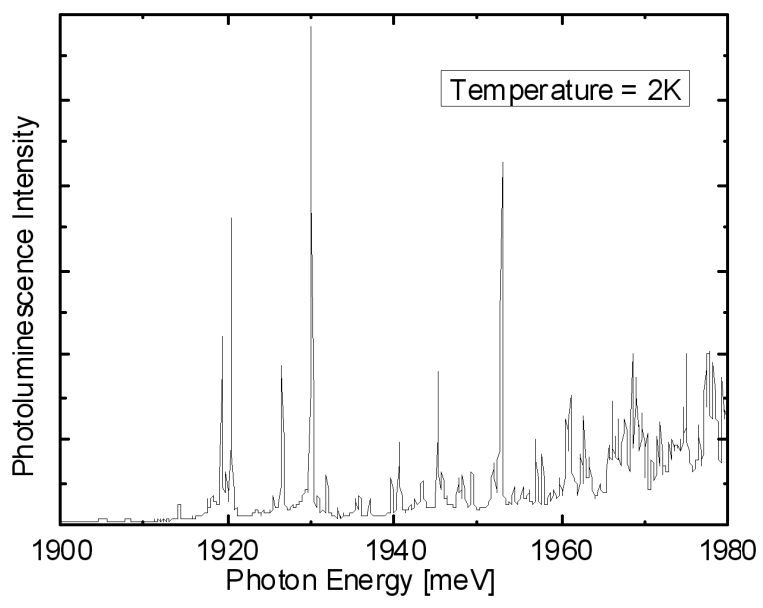

Fig. 1. The micro-photoluminescence spectrum measured for the QD sample.

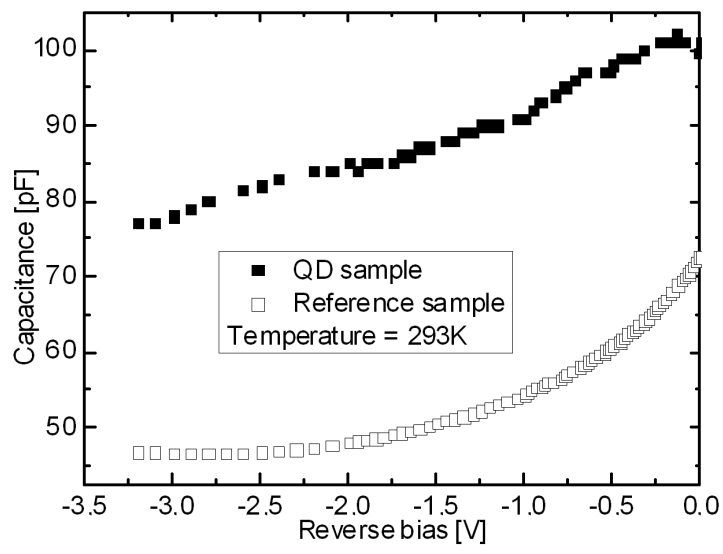

Fig. 2. $\quad C-V$ characteristics taken at $293 \mathrm{~K}$ for the sample with QDs (full squares) and for the reference sample (empty squares).

DLTS measurements were performed at temperatures in the range from $77 \mathrm{~K}$ to $300 \mathrm{~K}$ and at different lock-in frequencies under dark conditions. Figure 3a demonstrates a typical DLTS temperature scan for the reference sample which was performed at reverse bias equal to $-2 \mathrm{~V}$, with the filling pulse $V_{1}=0 \mathrm{~V}$ of $20 \mu$ s width and at a lock-in frequency equal to $28 \mathrm{~Hz}$. The DLTS temperature scan for the QD sample, taken at a lock-in frequency equal to $2.5 \mathrm{~Hz}$, reverse bias equal to $-2 \mathrm{~V}$, and the filling pulse $V_{1}=0.5 \mathrm{~V}$ of $20 \mu$ s width, is shown in Fig. 3b.

DLTS measurements for both samples reveal the presence of a broad high-temperature maximum that is much more pronounced for the QD sample. In order to distinguish the signal related to single traps the DLTS peaks have been simulated by separated Gaussian components [7]. The apparent activation energy $(E)$, its broadening $(\Delta E)$ and capture cross-section $(\sigma)$ were used as the fitting parameters. The apparent activation energy is the energy distance of a trap level (QD state) from the bottom of the ZnTe valence band and the capture cross-section determines the probability of a hole capture by a trap.

The values are as follows for the reference- and QD sample, respectively: $E_{\mathrm{H} 1 \mathrm{Ref}}=0.37 \mathrm{eV}, \sigma_{\mathrm{H} 1 \mathrm{Ref}}=1 \times$ $10^{-18} \mathrm{~cm}^{2} ; E_{\text {H2Ref }}=0.48 \mathrm{eV}, \sigma_{H 2 \text { Ref }}=8.8 \times 10^{-18} \mathrm{~cm}^{2}$ and $E_{\text {H3Ref }}=0.7 \mathrm{eV}, \sigma_{\text {H3Ref }}=1.36 \times 10^{-14} \mathrm{~cm}^{2}$, $E_{\mathrm{H} 1 \mathrm{QD}}=0.372 \mathrm{eV}, \sigma_{H 1 Q D}=1 \times 10^{-18} \mathrm{~cm}^{2} ; E_{\mathrm{H} 2 \mathrm{QD}}=$ $0.485 \mathrm{eV}, \sigma_{\mathrm{H} 2 \mathrm{QD}}=7 \times 10^{-18} \mathrm{~cm}^{2}$ and $E_{\mathrm{H} 3 \mathrm{QD}}=0.761 \mathrm{eV}$, $\sigma_{\mathrm{H} 3 \mathrm{QD}}=1.4 \times 10^{-14} \mathrm{~cm}^{2}$. The broadening was found to be around $10-30 \mathrm{meV}$.

For both samples observed DLTS maximum can be deconvoluted into three independent components having their origin in different autonomous defects. However the thermal activation energies and capture cross-sections obtained from the fitting are approximate for both samples. It can be concluded that the same defects are present in both samples. The difference is in the concentration of defects. The presence of QD CdTe layer magnifies their concentration as compared to the concentration of defects in the ZnTe matrix.

For both samples a positive peak appears on the DLTS scans in the temperature of $\approx 130 \mathrm{~K}$. Positive DLTS signal should not be observed for a perfect Schottky diode. It may appear due to a measurement artifact when a high frequency modulation $\omega$ is used and series resistance $R$ is high enough so that $R^{2} C^{2} \omega^{2} \gg 1[9]$. It may be also associated with surface states present at the metal-semiconductor interface. If the density of surface states is high than inversion layer underneath the metal electrode may be created and a hole capture can take place instead of a hole emission. In this case positive, minority-like, DLTS signal may occur. It was found that the aforementioned positive DLTS signal is the greater the more positive becomes the filling pulse $V_{1}$. Such a behavior indicates that the defects related to the signal are located at the metal-ZnTe interface. Thus it seems that the surface states are responsible for the positive DLTS signal in studied samples. Moreover the ideality factors for the diodes are significantly greater than 1 (2.3 for the reference sample and 4.9 for the QD sample) which further confirms this statement.

The direct band gap for ZnTe is $2.26 \mathrm{eV}$ and for CdTe - $1.49 \mathrm{eV}$. It is well known that the valence band offset for the $\mathrm{CdTe} / \mathrm{ZnTe}$ heterostructure interface exhibits the type II of alignment. CdTe valence band is located $0.1 \pm 0.06 \mathrm{eV}$ above $\mathrm{ZnTe}$ valence band $[10,11]$. However, it may be expected that the interface of the quantum dots CdTe embedded in ZnTe matrix would exhibit different properties for the strain accompanying QDs formation [12]. On the other hand, it may be assumed that the activation energy of a hole emission from the QDs to the ZnTe matrix valence band would not exceed several tens of meV. Unfortunately, this means that related DLTS signal can appear around- and at temperatures less than $100 \mathrm{~K}$, uncomfortably close to the apparent minority-like positive signal. 

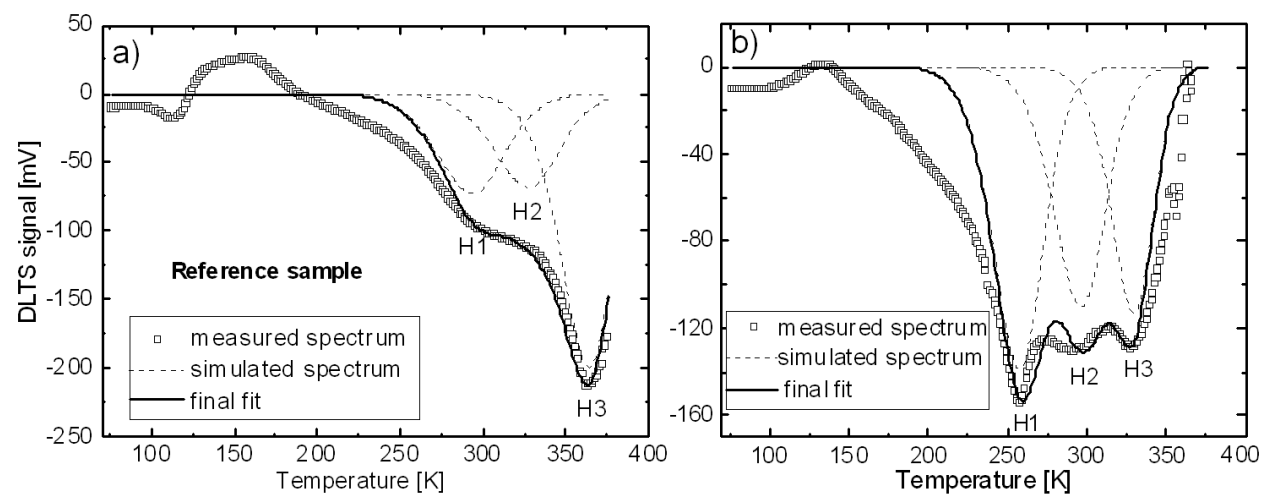

Fig. 3. Measured and simulated DLTS spectra for the reference (a) and QD sample (b). Dashed lines stand for the individual contributions simulated by separated Gaussians and solid lines - for the resultant net simulated spectra.

For now it may be concluded that the density of defects already present in ZnTe matrix significantly increases after QD formation. Such increase has been reported by many authors studying QD systems and has been assigned to the strain accompanying their creation $[13,14]$. No traps directly related to the QD hole confinement were observed for the studied CdTe/ZnTe system. Present result does not deny however possibility of hole confinement in $\mathrm{CdTe} / \mathrm{ZnTe}$ valence band. The presence of a high density of surface states in the studied samples leads to ambiguous result. In order to clarify the situation further studies on samples carefully prepared with less density of surface states are necessary.

\section{Conclusions}

The object of the investigations was a sample with CdTe quantum dots (formed by a Zn-induced reorganization of a thin CdTe layer) embedded in undoped ZnTe matrix. For a comparison a reference sample, in which QDs were absent, has been also examined. Both samples were investigated by means of the $C-V$ measurements and DLTS. The $C-V$ characteristic does not exhibit a step corresponding to the discharging of QDs whereas the micro-photoluminescence measurements exhibit QD behavior. The DLTS measurements for both samples yield the presence of three deep levels which have the origin in different autonomous defects. It was concluded that the same defects are present in both samples but there is an increased concentration of the defects in the QD structures. The latter seems to be a product of the strain present in the CdTe/ZnTe samples. No deep level associated directly with the QD confinement has been identified preasumably due to the presence of high density of surface states at a metal-ZnTe interface.

\section{References}

[1] H. Saito, K. Nishi, I. Ogura, S. Sugou, Y. Sugimoto, Appl. Phys. Lett. 69, 3140 (1996).

[2] D. Bimberg, N. Kirstaedter, N.N. Ledenstov, Zh.I. Alferov, P.S. Kop'ev, V.M. Ustinov, IEEE J. Sel. Top. Quantum Electron. 3, 196 (1997).

[3] P. Bhattacharya, K. Kamath, J. Singh, D. Klotzkin, J. Phillips, H. Jiang, N. Chevela, T. Norris, T. Sosnowski, J. Laskar, M. Ramanamurty, IEEE Trans. Electron Dev. 46, 871 (1999).

[4] D. Bimberg, M. Grundmann, N.N. Ledenstov, Quantum Dot Heterostructures, Wiley, Chichester 1998.

[5] H. Drexler, D. Leonard, W. Hansen, J.P. Kotthaus, P.M. Petroff, Phys. Rev. Lett. 73, 2252 (1994).

[6] S. Anand, N. Carlsson, M.E. Pistol, L. Samuelson, W. Seifert, J. Appl. Phys. 84, 3747 (1998).

[7] K. Dmowski, B. Lepley, E. Losson, M. El Bouabdellati, J. Appl. Phys. 74, 3936 (1993).

[8] C. Kruse, M. Gartner, A. Gust, D. Hommel, Appl. Phys. Lett. 90, 221102 (2007).

[9] A. Broniatowski, A. Blosse, P.C. Srivastava, J.C. Bourgoin, J. Appl. Phys. 54, 2907 (1983).

[10] T.M. Duc, C. Hsu, J.P. Faurie, Phys. Rev. Lett. 58, 1127 (1987).

[11] A. Continenza, S. Massidda, Phys. Rev. B 50, 11949 (1994).

[12] L. Klopotowski, M. Goryca, T. Kazimierczuk, P. Kossacki, P. Wojnar, G. Karczewski, T. Wojtowicz, Appl. Phys. Lett. 96, 201905 (2010).

[13] M. Kaniewska, O. Engstrom, A. Barcz, C.M. Pacholak, Mater. Sci. Eng. C 26, 871 (2006).

[14] J.S. Wang, J.F. Chen, J.L. Huang, P.Y. Wang, X.J. Guo, Appl. Phys. Lett. 77, 3027 (2000). 